\title{
Stiff man syndrome in a black girl
}

\author{
H Y A M I S A A C S \\ From the Clinical Neuromuscular Research Laboratory, Department of Physiology, \\ Witwatersrand University Medical School, Johannesburg, South Africa
}

SUMMARY A patient suffering from severe and continuous muscular spasms is presented. The condition has been classified as the stiff man syndrome and is the first case recorded of the condition in a young African girl. The electrophysiological, biochemical, histochemical, and ultramicroscopic aspects of this disorder have been studied. The spasm appears to be related to overproduction of brain noradrenaline.

In 1956 Moersch and Woltman published a paper in which they described a male patient of 49 years who exhibited a combination of fluctuating muscular rigidity and spasm. They named the condition the stiff man syndrome and at the same time reported their findings on 13 patients with similar complaints. Of the 13 , four were females, and it was thus no surprise when in 1958 Asher with his accomplished literary style and humour, published his paper on "A woman with stiff man syndrome". As the average age of onset of these patients was approximately 40 years, we thought that it would be of interest to present this particular patient because of her youth and the exceptionally severe state of muscular spasm. It is the first report of the condition occurring in a young girl and in a black patient of African parentage.

\section{Case report}

The patient, an 8 year old black girl (Fig. 1), gave a history of progressive disability which extended over a period of 18 months. The condition, as far as could be determined, began with muscle stiffness and spasms which affected the trunk and proximal muscles of upper and lower extremities. This slowly spread to the peripheral musculature. The severity of the spasms gradually increased up to the present. The child is now in constant pain and totally immobilised by her illness.

No family history of neuromuscular disease could be obtained, and there was no history of any

Address for reprint requests: Dr H. Isaacs, Clinical Neuromuscular Research Laboratory, Department of Physiology, Witwatersrand University Medical School, Hospital Street, Hospital Hill 2001, Johannesburg, South Africa.

Accepted 17 April 1979 serious disease or of surgery in the past. Intellectual development was normal.

On physical examination the child was found to be lying in a position of oposthotonos. The spasms affected the muscles of the back, trunk, and extremities in order of severity. The spasms were continuous during her waking state and were्ष aggravated by external stimuli, such as skin con $\cong$

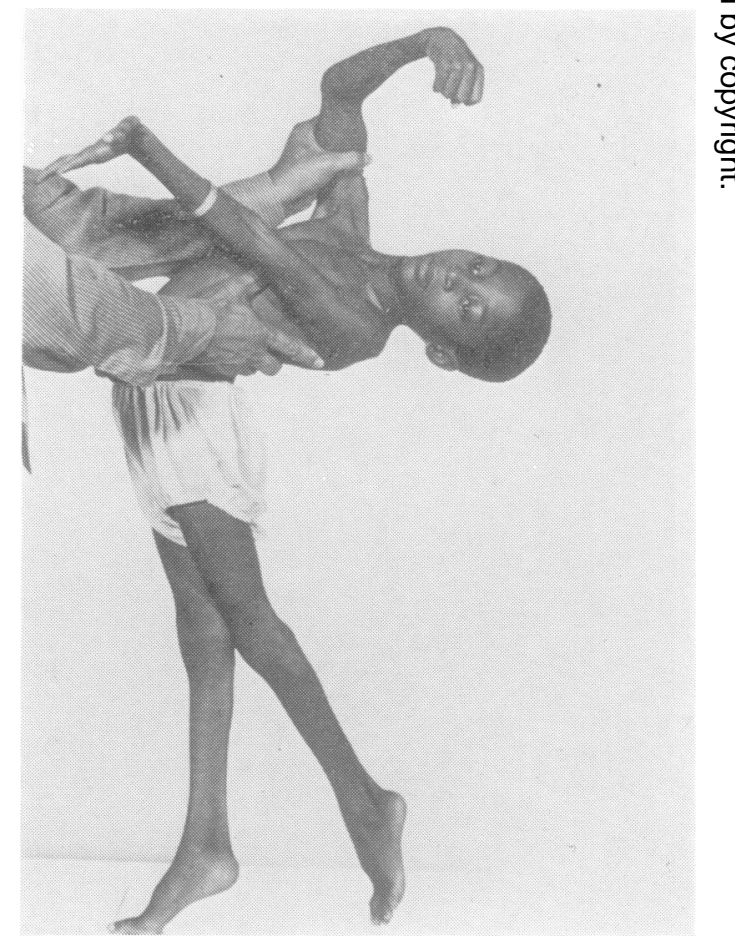

Fig. 1 The patient. 
tact, emotional stimuli, and movement of the limbs both active and passive. There was no evidence of any sensory defect. Though difficult to test, coordination and muscle strength appeared to be normal. The hands were held in a flexed position with the thumbs, for the most part, being strenuously opposed to the index and third fingers. The facial and oropharyngeal muscles and the muscles of mastication were not involved. The tendon jerks were difficult to elicit because of the state of muscular spasm. The plantar responses appeared to be normal, though again these were difficult to elicit as stroking the skin of the soles and abdomen induced severe spasm.

\section{INVESTIGATIONS}

The usual routine metabolic and endocrine investigations were completely normal. The patient was mildly pyrexial throughout her stay in hospital. This was related to the continuous muscle activity and tended to subside at the times when her spasms were less. Her blood pressure was $110 / 70$ $\mathrm{mmHg}$, the pulse was regular and the rate averaged $100 /$ minute. The Valsalva response was normal and there was no sign of postural hypotension. Sweating and sphincteric control were normal. The ECG and EEG were normal. Cerebrospinal fluid analysis was normal. Radiographs of the patient's chest and skeletal films, apart from a spina bifida affecting the lower lumbar segments, were normal.

Electrophysiological study revealed spontaneous motor unit activity of normal potential amplitude and duration involving both agonists and antagonists at rest. This activity was aggravated by emotional disturbance, touch, and movement. Full recruitment pattern was present on effort, and the voluntary muscle contraction caused diffusion of contraction to all muscles, both agonists and antagonists. There was no denervation activity, conduction velocities in both motor and sensory nerve fibres were normal. Silent periods in muscles provoked by nerve stimulation were normal. Blocking of the peripheral nerve with procaine caused flaccid paralysis with absence of motor unit activity, and the myoneural junction blocked normally with pancuronium. The $\mathrm{H}$ reflex was difficult to suppress with vibration. Polysynaptic nonpainful reflexes were abnormal in that there was no evidence of habituation and co-contraction occurred in antagonistic muscles.

Muscle and nerve biopsies from the left vastus lateralis were carried out under general anaesthesia and a section of sural nerve was obtained. A strip of muscle was subjected to tension studies, and the response to halothane and varying con- centrations of caffeine was normal. The muscle histology and histochemistry was within normal limits (Figs. 2, 3). Electronmicroscopy revealed areas of myofilamentous and Z-line disruption (Fig. 4) as well as mitochondrial accumulation. Many of the mitochondria were distended and the cristae ruptured (Fig. 5). The appearance of the sural nerve was normal (Fig. 6).

Specific studies on neurotransmitters were performed by estimations of urinary excretion. The usual precautions with regard to diet were adopted for at least 48 hours before vallinyl mandelic acid (VMA) collections which were assayed by the method of Dekirmenjian and Maas (1970). As a means of assessing dopamine activity, urinary homovanillic acid (HVA) was assayed according to the method of Dziedzic et al. (1972). Metanephrine (ME) and normetanephrine (NME), the excretory products of epinephrine (adrenaline) and norepinephrine (noradrenaline) respectively were assayed by the method of Taniguchi et al. (1964). 3 Methoxy-4-hydroxy-phenyl glycol (MHPG) was assayed according to the method of

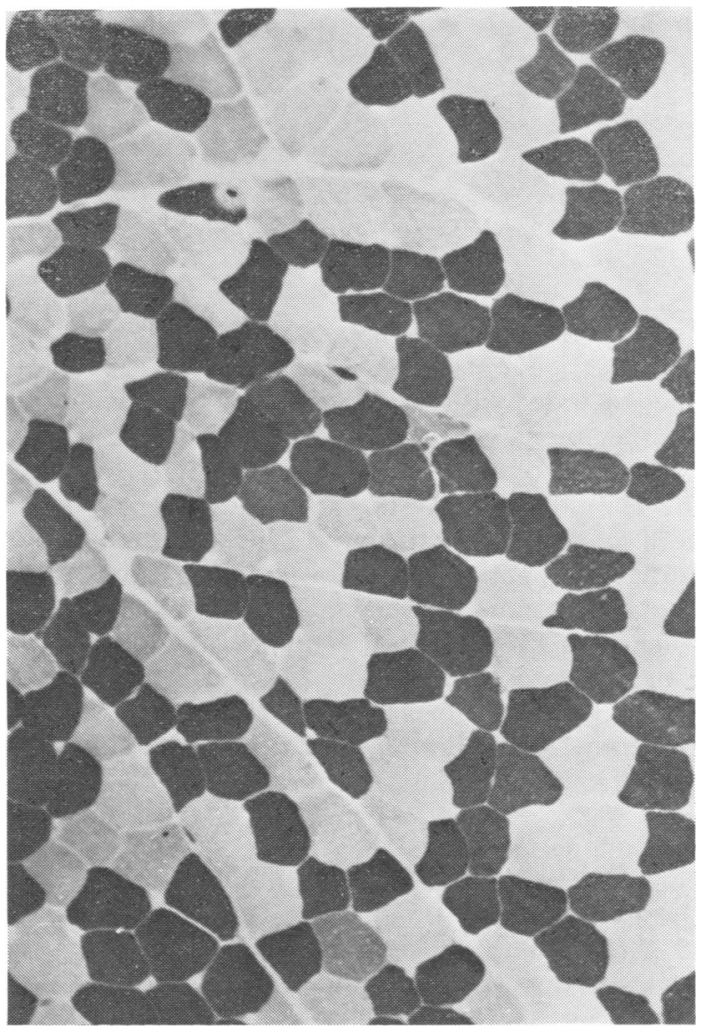

Fig. 2 Normal mitochondrial ATPase stain at $\mathrm{pH}$ 4.6 , original magnification $\times 300$. 


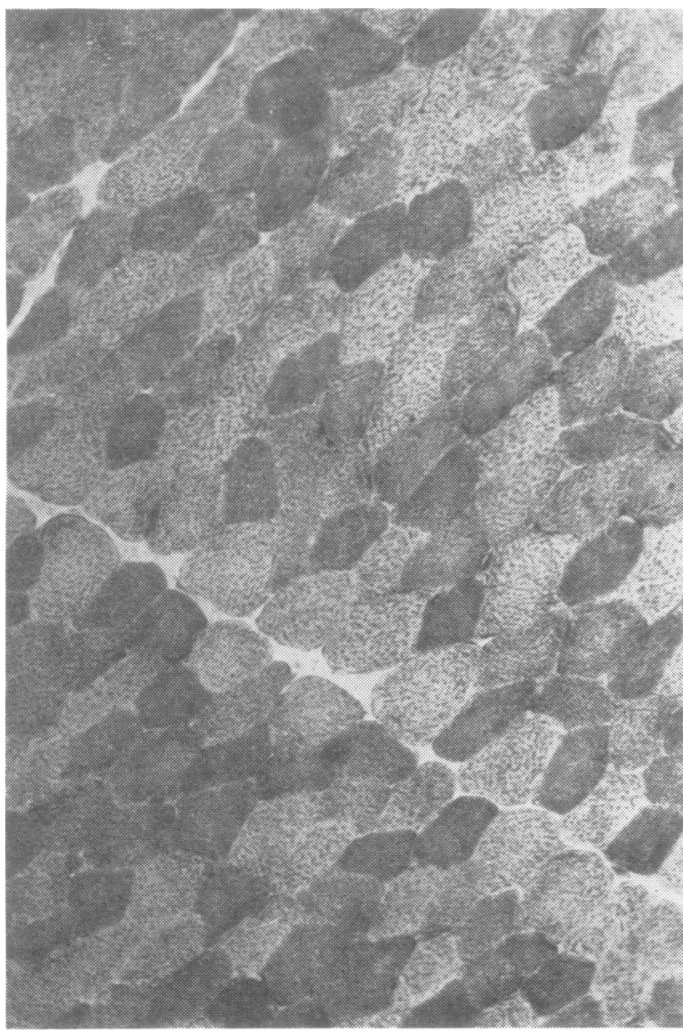

Fig. 3 Normal NADH stain, original magnification $\times 300$.

Bonsnes and Taussky (1945). MHPG estimations were carried out as this substance is reputed to give an index of brain noradrenaline production. All specimens were correlated with renal function by way of creatinine clearance: VMA, ME, NME, and HVA levels were normal. The excretory levels were matched with those of a healthy inpatient of similar age and sex who was recovering from a fractured thigh. The present patient's urine contained MHPG $1,950 \pm 105 \mathrm{mg} / 24 \mathrm{hr}$. The control patient produced $1,406 \pm 84 \mathrm{mg} / 24 \mathrm{hr}$. Our patient's MHPG levels were higher than the levels quoted by Schmidt et al. (1975) for normal females at $1,674 \pm 117 \mathrm{mg} / 24 \mathrm{hr}$.

\section{THERAPEUTIC RESPONSE}

The patient did not respond clinically to oral glycine administration in a divided daily dose of $15 \mathrm{~g}$ though this increased the serum level from 2.1 to $32 \mathrm{mg} / \mathrm{dl}$. Pyridostigmine bromide in a dose of $60 \mathrm{mg}$ six hourly caused no change in the muscle spasm over three days. Diphenylhydantoinate $100 \mathrm{mg}$ three times daily failed to relieve the spasm over three days. Diazepam given in increasing dosage up to $60 \mathrm{mg} /$ day had a beneficial effect, and the drug was well tolerated. The addition of clonazepam $0.5 \mathrm{mg}$ four times daily seemed to make little difference.

On a reasonably spasm-free day on diazepam, the MHPG levels had dropped to $1,364 \pm 76 \mathrm{mg} /$ $24 \mathrm{hr}$. MHPG was raised to $2,100 \pm 66 \mathrm{mg} / 24 \mathrm{hr}$, and the spasms aggravated by the administration of L-dopa $125 \mathrm{mg}$ four times daily. Baclofen (Lioresal) in a dose of $10 \mathrm{mg}$ three times daily seemed to be as effective as diazepam but induced nausea. The urinary level of MHPG on one occasion on baclofen fell to $806 \pm 26 \mathrm{mg} / 24 \mathrm{hr}$.

Physiotherapy was started and the contractures were slowly beginning to lengthen when the parents removed the child from hospital, and she has not been seen or heard of since.

\section{Discussion}

The stiff man syndrome as described by Moersch and Woltman (1956) may deteriorate to a point where the patient's body may be distorted by painful continuous muscle spasm. The spasms, as $\infty_{\infty}^{\infty}$ in our patient, increased in severity in respons 8 to sensory or emotional stimuli and attempts to move the muscles either actively or passivelo $z$ evoked a most painful reaction. The spasms are involuntary in nature and are seen as violent an painful contractions in both agonists and antagon ists. In most cases the proximal muscles are ine volved earlier and more severely than the distaf, $\vec{\oplus}$ and the legs tend to be involved more than the 0 arms. The ultimate posture of the body is severely distorted because of the pull of the more powerful muscles. The abnormal muscle activity makes walking impossible and the patient tends to fall and, to quote from Moersch and Woltman, "falls like a wooden man" or "like a wax dummy." They are unable to sit because the trunk and thighs constitute what was described by Martinelli et al. (1978) as "a continuous single block." Respiratory insufficiency may become a problem at times when the muscle stiffness and pain tend to interfere with normal respiration. The facial, oculopharyngeal, and mastication muscles are, for some unexplained reason, unaffected.

Very little is known of the pathology of stiff man syndrome. Two of the original cases of $₹$ Moersch and Woltman were subjected to muscle 웅 biopsy with normal results. A postmortem examination carried out on another of their patients revealed no abnormality in the brain but unfortunately the spinal cord was not examined. Detailed pathological examinations carried out by 


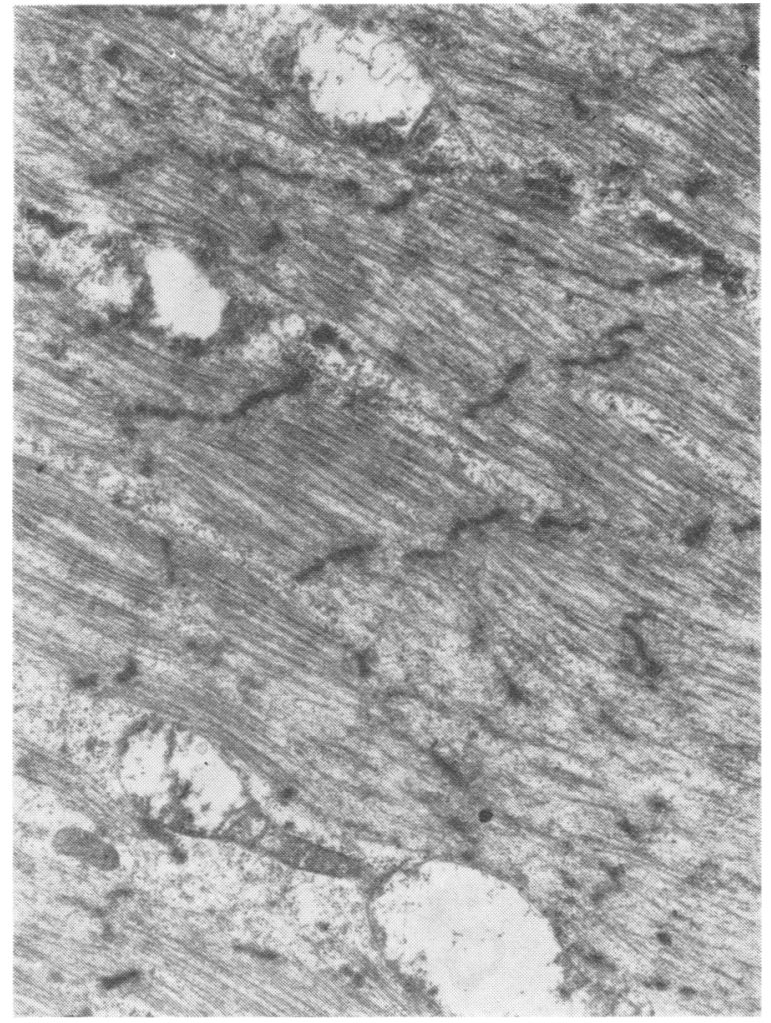

Asher (1958), Trethowan et al. (1960), and Cohen (1966) were reported as normal. The myofilamentous disruption in our case may be attributed to the violent muscle spasms though the mitochondrial changes, apart from artefact which is unlikely, are unexplained and not related to the mitochondrial myopathy of Luft et al. (1962).

It is significant that the incidence of epilepsy in patients suffering from stiff man syndrome is $10 \%$. In addition several cases of stiff man syndrome have exhibited another manifestation of spontaneous neurogenic discharge in the form of nocturnal myoclonus. Our case suffered neither nocturnal myoclonus nor epilepsy. Nocturnal myoclonus is usually found in association with both spinal and supraspinal lesions. The increased incidence of epilepsy and myoclonus if coupled with stiff man syndrome suggests a central source of neuronal irritability for the latter.

Results of electrophysiological studies as seen in our patient and others are fairly characteristic and are outlined in the Table. The overall interpretation of the electrophysiological evidence suggests a state of hyperexcitability at spinal level as exhibited by both the polysynaptic and monosynaptic
Fig. 4 Electronmicrograph showing filamentous depletion. Disruption of Z-lines and distended mitochondria. A few triads are visible, original magnification $\times 16000$.
Table Summary of electrophysiological features of the stiff man syndrome

Electrophysiological features

1 Spontaneous motor unit activity of normal amplitude and duration. Involving both agonists and antagonists at rest. Aggravated by fright, touch, movement

2 Full recruitment pattern on effort but contraction causes diffusion to all muscles

3 No denervation activity

4 Conduction velocities in both motor and sensory nerve fibres are normal

5 Silent period in the anterior tibial muscle provoked by stimulation of the peroneal nerve at the head of the fibula is normal

6 Blocking of the peripheral nerve with procaine causes flaccid paralysis

7 The $\mathrm{H}$ reflex is easily obtained with normal delays. Not suppressed by vibration

8 Polysynaptic nonpainful reflexes are abnormal in that there is no evidence of habituation and co-contraction occurs in antagonistic muscles

reflex systems. This in itself suggests that the motor neuronal activity is underdamped as a result of disturbed function of the suppressor system either at the spinal cord or at brain level. Abnormal activity at a muscular level was excluded by demonstrating paralysis after blockade of the 


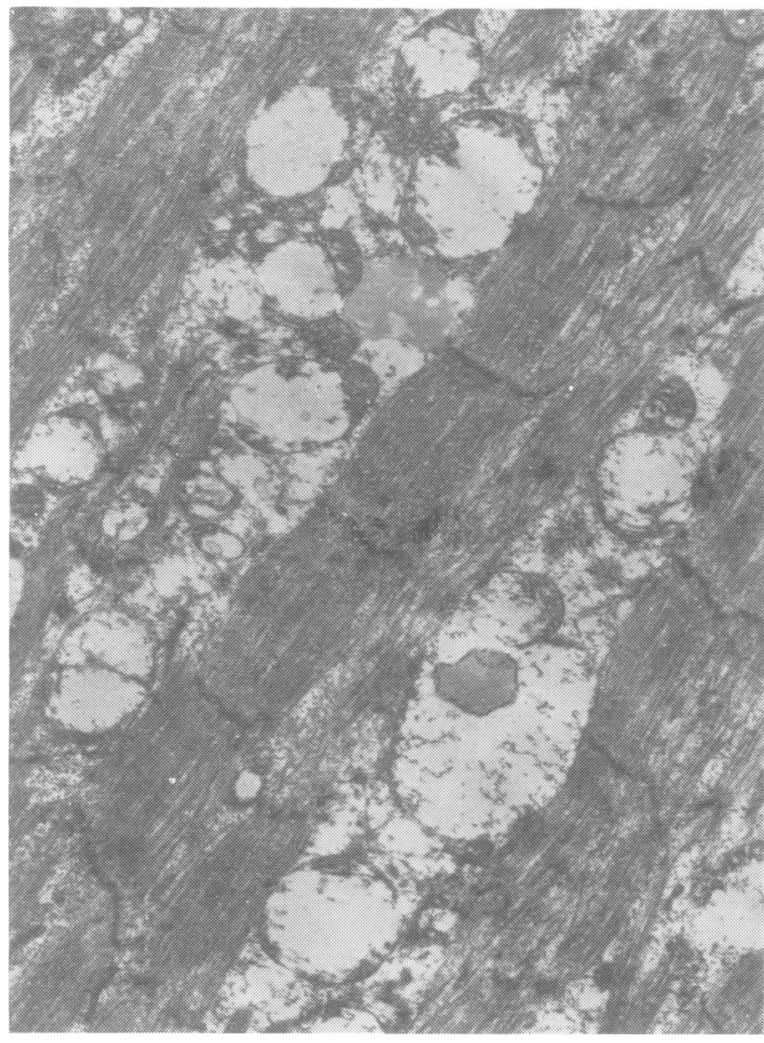

Fig. 5 Electronmicrograph showing distended mitochondria and filamentous depletion, original magnification $\times 1200$. myoneural junction. Furthermore, electromyography excluded the presence of myotonic activity, and Isaac's syndrome (1961) was excluded by the immediate disappearance of motor unit activity on peripheral nerve blockade at a proximal level. The absence of electromyographic evidence of muscle cramp excluded the syndrome of progressive muscle spasm with alopecia and diarrhoea (Satoyoshi, 1978) .

The Renshaw cell system suppresses excitability and overactivity of the motor neurones by reducing the rate of discharge, and in so doing prevents synchronous activity. These recurrent axon collaterals are acetylcholine-dependent and as such their activity is enhanced by neostigmine. As with the case of Olafson et al. (1964), our patient showed no response to large therapeutic doses of this acetylcholinesterase inhibitor, negating defective activity at this level.

The spasms of stiff man syndrome bear a marked similarity to strychnine poisoning where the hyperactive state is presumed to be caused by the lack of suppressive activity of the spinal glycinergic interneuronal system. Gelfan and Tarlov $(1959,1963)$ were able to produce experimentally a lesion in the glycinergic system by inducing spinal cord ischaemia. Christensen et al. (1947) demonstrated the beneficial effect of glycine on this type of muscle spasm and the hind limb rigidity in rats induced by ischaemia was shown to be relieved by glycine administration (Stem and Hadzovic, 1970). Schmidt et al. (1975) used glycine in the treatment of stiff man syndrome without success, and our patient was given oral glycine in a dose of $15 \mathrm{~g}$ daily on three consecutive days without clinical improvement. These results support the findings of Schmidt et al. (1975) in establishing that it is unlikely that the glycinergic spinal inhibitory interneuronal system is responsible for the neuronal malfunction in stiff man syndrome unless this system be so pathologically damaged as to be unresponsive. This hypothesis is negated by the absence of pathological findings and by the clinical response observed with other pharmacological agents.

The dopamine and peripheral catecholinergic systems were considered normal on the basis of excretory studies involving HVA, ME, NME, and VMA-ME, NME, and VMA are derived from peripheral adrenaline and noradrenaline, with 


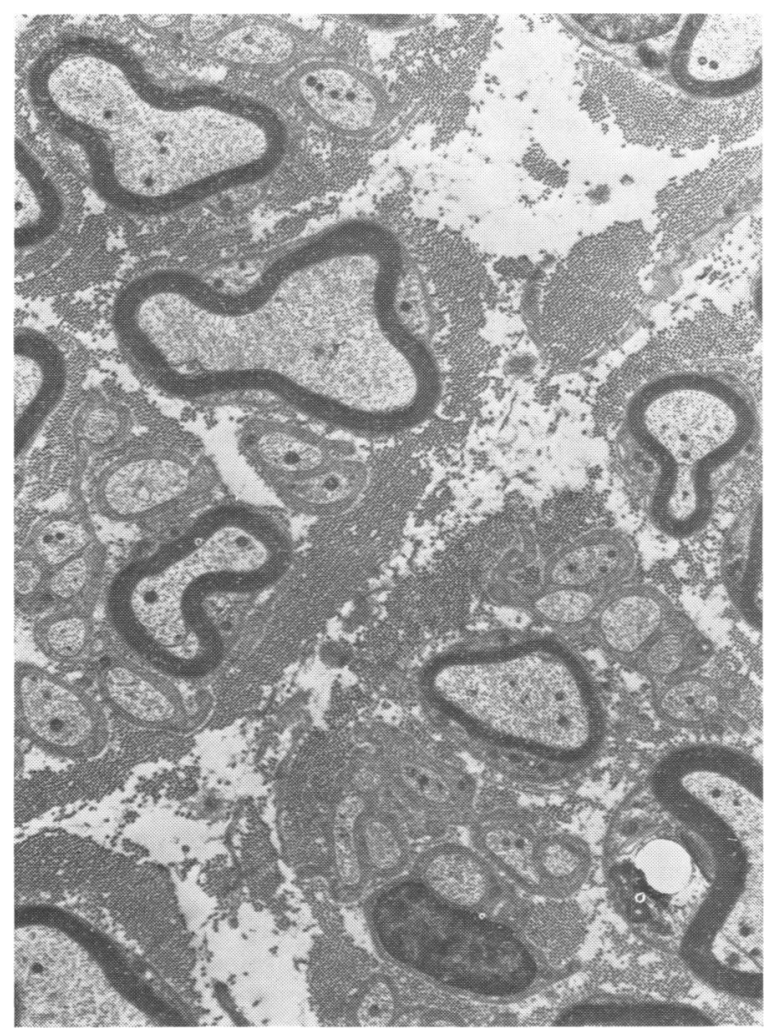

Fig. 6 Cross-section of sural nerve showing normal myelinated and nonmyelinated fibres, original magnification $\times 6000$.

HVA being the excretory product of the dopamine system. MHPG derived from central or brain noradrenaline was raised. This is in contrast to the findings in Parkinson's disease where peripheral adrenaline of adrenal origin is increased and is reduced by the administration of levodopa (Stoica and Enulescu, 1978). Levodopa aggravated the spasms of stiff man syndrome and is known to lower brain concentration of S-adenosylmethionine. This substance is necessary for the transfer of methyl groups in the formation of adrenaline from noradrenaline (Chalmers et al., 1971) thus reducing adrenaline formation and increasing noradrenaline concentration. This alteration may account for the deterioration of the symptoms in stiff man syndrome on L-dopa.

The therapeutic effect of diazepam in the stiff man syndrome was described by Howard (1963), and this has helped with the localisation of the dysfunction. Diazepam reduces the level of excitation of both spinal and brain catecholaminergic neurones and increases the levels of gamma aminobutyric acid (GABA). Baclofen, a centrally active GABA derivative, has some effect in reducing spasticity and also reduces the intensity of the spasms in stiff man syndrome. Underactivity of the GABA system as the basic defect in the stiff man syndrome is largely negated, however, by the absence of response to the administration of dipropylacetate. Dipropylacetate increases GABA concentration in the brain (Schmidt et al., 1975). This investigation confirms the work of Schmidt et al. (1975) in producing evidence of catecholaminergic overactivity by way of increased MHPG excretion and the modification of excretion by various therapies coupled with the clinical response.

This work was carried out in the Clinical Neuromuscular Research Laboratory of the Department of Physiology at the University of the Witwatersrand with the aid of grants from Medical Research Council, The Muscular Dystrophy Research Foundation of South Africa, and the University of the Witwatersrand.

\section{References}

Asher, R. (1958). A woman with stiff-man syndrome. British Medical Journal, 1, 265-266. 
Bonsnes, R. W., and Taussky, H. H. (1945). On the colorimetric determination of creatinine by the Jaffa reaction. Journal of Biological Chemistry, 158, 581-591.

Chalmers, J. P., Baldessarini, R. J., and Wurtman, R. J. (1971). Effects of L-dopa on norepinephrine metabolism in the brain. Proceedings of the National Academy of Sciences of the United States of America, 68, 662-666.

Christensen, H. N., Cooper, P. F., Johnson, R. D., and Lynch, E. L. (1947). Glycine and alanine concentrations of body fluids: experimental modification. Journal of Biological Chemistry, 168, 191-196.

Cohen, L. (1966). Stiff man syndrome: two patients treated with diazepam. Journal of the American Medical Association, 195, 222-224.

Dekirmenjian, H., and Maas, J. (1970). An improved procedure of 3-methoxy-4-hydroxy-phenylglycol determination by gas-liquid chromatography. Annals of Biochemistry, 35, 113-122.

Dziedzic, S. W., Bertani, L. N., Clarke, D. D., and Gitlow, S. E. (1972). A new derivative for the gasliquid determination of homovanillic acid. Analytical Biochemistry, 47, 592-600.

Gelfan, S., and Tarlov, I. M. (1959). Interneurones and rigidity of spinal origin. Journal of Physiology, 146, 594-617.

Gelfan, S., and Tarlov, I. M. (1963). Altered neuronal population in $\mathrm{L} 7$ segment of dogs with experimental hind-limb rigidity. American Journal of Physiology, 205, 606-616.

Howard, F. M. (1963). A new and effective drug in the treatment of stiff-man syndrome: preliminary report. Proceedings of the Staff Meetings of the Mayo Clinic, 38, 203-212.

Isaacs, H. (1961). A syndrome of continuous musclefibre activity. Journal of Neurology, Neurosurgery, and Psychiatry, 24, 319-325.

Luft, R., Ikkos, D., Palmieri, G., Ernster, L., and Afzelius, B. (1962). A case of severe hypermeta- bolism of nonthyroid origin with a defect in the maintenance of mitochondrial respiratory control: a correlated clinical biochemical and morphological study. Journal of Clinical Investigation, 41, 17761804.

Martinelli, P., Pazzaglia, P., Montagna, P., Coccagna, G., Rizzuto, N. Simonati, S., and Lugaresi, E. (1978). Stiff-man syndrome associated with nocturnal myoclonus and epilepsy. Journal of Neurology, Neurosurgery, and Psychiatry, 41, 458-462.

Moersch, F. P., and Woltman, H. W. (1956). Progressive fluctuating muscular rigidity and spasm ("stiff-man" syndrome): report of a case and some observations in 13 other cases. Proceedings of the Staff Meetings of the Mayo Clinic, 21, 421-427.

Olafson, R. A., Mulden, D. W., and Howard, F. M. (1964). "Stiff-man" syndrome: a review of the literature, report of three additional cases and discussion of pathophysiology and therapy. Mayo Clinic Proceedings, 39, 131-143.

Satoyoshi, E. (1978). A syndrome of progressive muscle spasm, alopecia and diarrhoea. Neurology (Minneapolis), 28, 458-471.

Schmidt, R. T., Stahal, S. M., and Spehlmann, H. R. (1975). A pharmacologic study of the stiff man syndrome Neurology (Minneapolis), 25, 622-626.

Stem, P., and Hadzovic, S. (1970). Effect of glycine 0 on experimental hind-limb rigidity in rats. Life्ष Sciences, 9, 955-959.

Stoica, E., and Enulescu, O. (1978). Abnormal epine phrine urinary excretion in Parkinsonians-correc tion of the disorder by levodopa administration Journal of the Neurological Sciences, 38, 215-227.

Taniguchi, K., Kakimoto, Y., and Armstrong, M. D (1964). Quantitative determination of metanephrin and normetanephrine in urine. Journal of Labora tory and Clinical Medicine, 64, 469-484.

Trethowan, W. H., Allsop, J. L., and Turner, B. (1960). The "stiff-man" syndrome. Archives of Neurology (Chicago), 3, 448-456. 